\section{Cureus}

Received 12/04/2014

Review began 12/06/2014

Review ended 03/02/2015

Published 03/20/2015

\section{(c) Copyright 2015}

Angus et al. This is an open access article distributed under the terms of the Creative Commons Attribution License CC-BY 3.0., which permits unrestricted use, distribution, and reproduction in any medium, provided the original author and source are credited.

\title{
Asthma Exacerbation: An Emergency Medicine Simulation Scenario
}

\author{
Karen Angus $^{1}$, Michael Parsons ${ }^{2}$, Neil Cheeseman ${ }^{1}$, Adam Dubrowski ${ }^{3}$ \\ 1. Discipline of Emergency Medicine, Memorial University of Newfoundland 2. Emergency Medicine, \\ Memorial University of Newfoundland 3. Emergency Medicine, Pediatrics, Memorial University of \\ Newfoundland
}

$\square$ Corresponding author: Adam Dubrowski, adam.dubrowski@gmail.com Disclosures can be found in Additional Information at the end of the article

\section{Abstract}

In the practice of emergency medicine, simulation is a valuable tool that allows medical students and postgraduate residents to develop skills in a safe environment at no risk to patients. In this report, we present a case simulation of an acute asthma exacerbation utilizing a human patient simulator. The case is designed such that it can be easily modified to accommodate the trainee's level of expertise, allowing instructors to challenge both the novice and advanced learner alike.

Categories: Emergency Medicine, Medical Simulation

Keywords: emergency medicine, simulation based medical education, asthma exacerbation, simulation

\section{Introduction}

Asthma exacerbation is a problem frequently encountered in the emergency department. Its severity at presentation can range from a mild increased shortness of breath to respiratory arrest and death. In 2009, it accounted for 2.1 million emergency department visits in the United States alone [1]. Although asthma fatalities have steadily declined since the 1990s, the early recognition and treatment of asthma exacerbation is vital to preventing patient deterioration and possible death [1-2].

The biggest challenge in treating asthma exacerbation lies in identifying patients who are most at risk for deterioration and instituting prompt action. It has been suggested that there exists a window of opportunity wherein aggressive treatment of such a deteriorating patient may prevent a fatal outcome [2].

Simulation has been shown to enhance knowledge and skills acquisition as well as improve patient outcomes in emergency medicine and for other health care learners [3]. This technical report details a simulation training session designed for a cohort of emergency medicine trainees in their third and final year of training at Memorial University of Newfoundland. Its objectives focus on identifying and managing the patient presenting with an acute asthma exacerbation utilizing basic treatments and thereafter progressing through a series of more aggressive interventions in response to patient deterioration. The scenario's stepwise design enables it to escalate in complexity, allowing its use in training a wide variety of learners, such as undergraduate medical students and residents in a family practice setting.

\section{Technical Report}




\section{Cureus}

This simulation session was conducted in a lab and utilized both a confederate voicing the role of the patient, as well as a high-fidelity mannequin simulator. Although a confederate may be used to play the patient throughout the scenario up to the point of requiring intubation, the progressive tachypnea demonstrated may be difficult for a confederate to simulate in an effective and safe manner.

Prior to the session, we developed a patient script as well as a stepwise, detailed scenario template (Table 1). This process required collating all the information and supporting documents such as EKG/ECG tracings (Figure 1) and x-rays (Figures 2, 3) that were to be used during the scenario execution. These templates were then submitted to the simulation lab's technical staff who reviewed the script, programmed the mannequin, and supplied required materials for the scenario's execution.

\section{Pre-Scenario}

You are working in a tertiary care emergency department with subspecialty backup available. A 23 y.o. female presents complaining of shortness of breath. She has been triaged and is now awaiting your assessment.

\section{History}

Allergies

Environmental, to dust and dander

Medications

Symbicort $^{\mathrm{TM}}$, Ventolin $^{\mathrm{TM}}$, finished prednisone 1 week ago

Past Medical $\mathrm{Hx}$

Asthma (past hospital / ICU admission)

Social $\mathrm{Hx}$

Smoker, $1 / 2$ ppd

Family Hx

Nil Significant

Initial Vitals

T 36.9 // HR 121 // RR 24 // BP 142/72 // Sat 90\% The patient is sitting on a gurney, alert but anxious.

HEENT

Oropharynx and TMs normal on exam

CNS

Normal apart from anxious appearance

Chest

Normal heart sounds; increased work of breathing with accessory muscle use noted; decreased breath sounds bilaterally

Abdomen

Benign

\section{Expected Actions}

Place patient on telemetry Obtain IV access Administer supplemental oxygen Order EKG (Fig. 1), labs/X-rays (Figs. 2, 3): electrolytes, CBC, BUN, Cr, glucose, ABG, CXR

\section{Objective 1: Managing An Acute Asthma Exacerbation}
Stage
Vitals
Expected Action

As the patient is being examined, she becomes more tachypneic with difficulty speaking in full

T36.9 ${ }^{\circ} \mathrm{C}$, HR 130, BP 104/74, RR 40 , Sat $87 \%$
Administer nebulized salbutamol or ipratropium/salbutamol combination

sentences. 


\section{Cureus}

If treated with oxygen and nebulized

medications

If no treatment other than oxygen and nebulized medications

If treated with oxygen, nebulized medications, steroids, and MgSO4
T $37^{\circ} \mathrm{C}$, HR 150 , BP $108 / 76$, RR 39 , Sats $85 \%$

T37 ${ }^{\circ} \mathrm{C}$, HR 160 , BP $100 / 70$, RR

44 , Sats $82 \%$

T37 ${ }^{\circ} \mathrm{C}$, HR 145, BP 108/76, RR Proceed to Objective 2. May also consider inhaled 39 , Sat $88 \%$
Administer steroids and MgSO4

Administer MgSO4 and/or steroids anesthetic agent or IM/ IV/inhaled epinephrine

Objective 2: Managing Respiratory Fatigue In Status Asthmaticus

The patient's respiratory

rate begins to slow

Respiratory exam

reveals minimal air entry

and no wheeze.

If PPV is not initiated
T $37^{\circ} \mathrm{C}$, HR 148 , BP $106 / 74$, RR 28 , Sat $87 \%$

Pt becomes progressively drowsy with decreased LOC
Re-examine the patient

Initiate positive pressure ventilation (BiPAP/CPAP)

Intubation

\section{Objective 3: Managing Respiratory Failure}

Stage

Vitals

Expected Actions

While on PPV, the patient develops drowsiness with confusion

T $37^{\circ} \mathrm{C}$, HR 136, BP 110/76, RR 16 (Vent Settings), Sats 83\% RR 16 (vent settings)- if intubated RR 24 - if on PPV

Respiratory exam reveals a silent chest (patient driven)

Recognize signs of respiratory failure; reexamine patient

Initiate rapid-sequence intubation using ketamine as preferred induction agent; confirm proper tube placement using at least 3 methods either observed or verbalized by learner (e.g. waveform capnography; CXR; auscultation, etc.)

\section{Objective 4: Managing Complications of RSI}

\begin{tabular}{|c|c|c|}
\hline Stage & Vitals & Expected Actions \\
\hline $\begin{array}{l}\text { Patient becomes } \\
\text { hypotensive post- } \\
\text { intubation }\end{array}$ & $\begin{array}{l}\text { T } 37^{\circ} \mathrm{C}, \text { HR } 110, \text { BP } 72 / 48, \\
\text { RR12 (Vent Settings) Sat } 96 \%\end{array}$ & Initiate fluid bolus(es) \\
\hline If fluid bolus(es) given & $\begin{array}{l}\text { T } 37^{\circ} \mathrm{C}, \mathrm{HR} 105, \mathrm{BP} 85 / 60 \text {, } \\
\text { Sats } 96 \% \text {. }\end{array}$ & $\begin{array}{l}\text { Considers breath stacking; checks ventilator rate/ } \\
\text { insp/exp ratio; considers tension pneumothorax as } \\
\text { cause; initiates vasopressors. }\end{array}$ \\
\hline If vasopressors started & $\begin{array}{l}\text { T37 }{ }^{\circ} \mathrm{C} \text { HR } 105, \text { BP } 102 / 72, \\
\text { Sats } 96 \%\end{array}$ & Consultation to ICU \\
\hline
\end{tabular}

Stabilization and transfer to ICU if: Initial treatments of asthma exacerbation are initiated Patient's respiratory fatigue 


\section{Cureus}

and subsequent respiratory failure is addressed Hypotension is addressed

TABLE 1: A stepwise, detailed scenario template provided to simulation laboratory technical staff. Figures 1 to 3 are part of Table 1 detailed scenario template.

(*Note that for a junior trainee, all vitals may be modified to reflect a more stable patient and scenario may be terminated after Objective 1 criteria are met*)

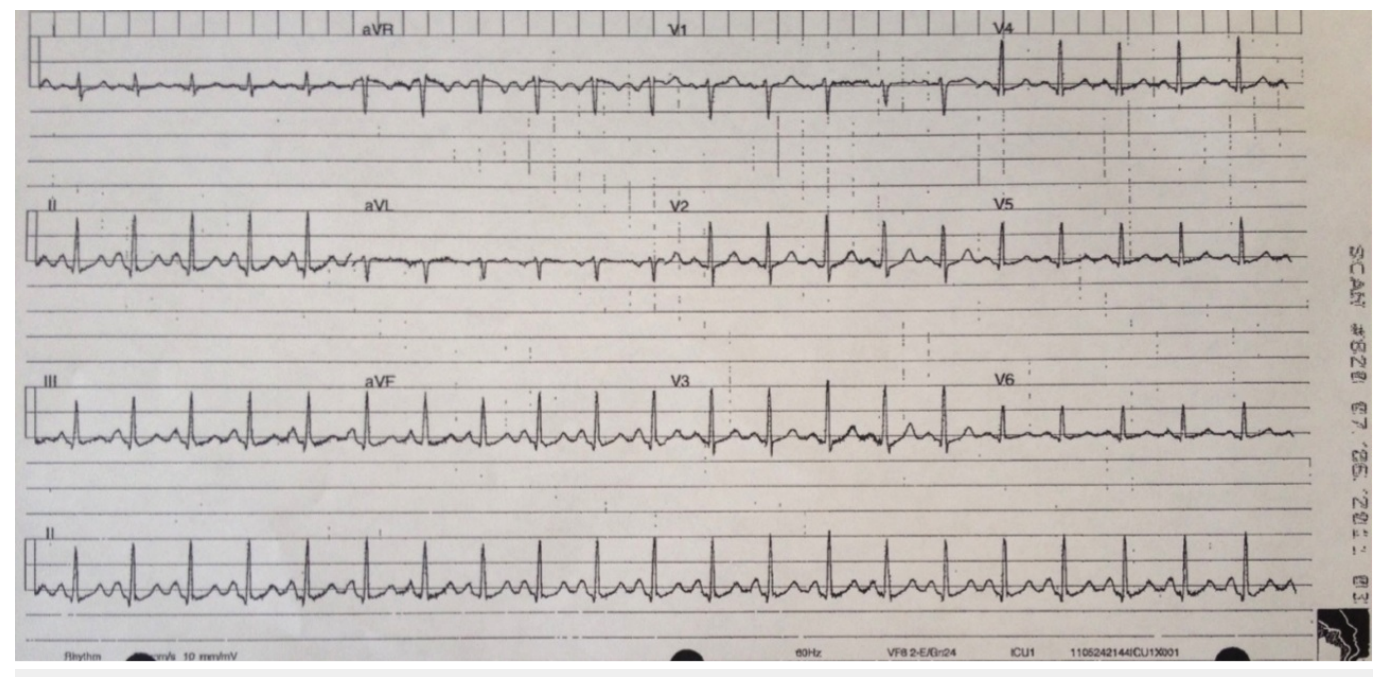

FIGURE 1: An electrocardiogram (EKG or ECG) demonstrating sinus tachycardia in a patient presenting with acute asthma exacerbation (Source: MH Parsons) 


\section{Cureus}

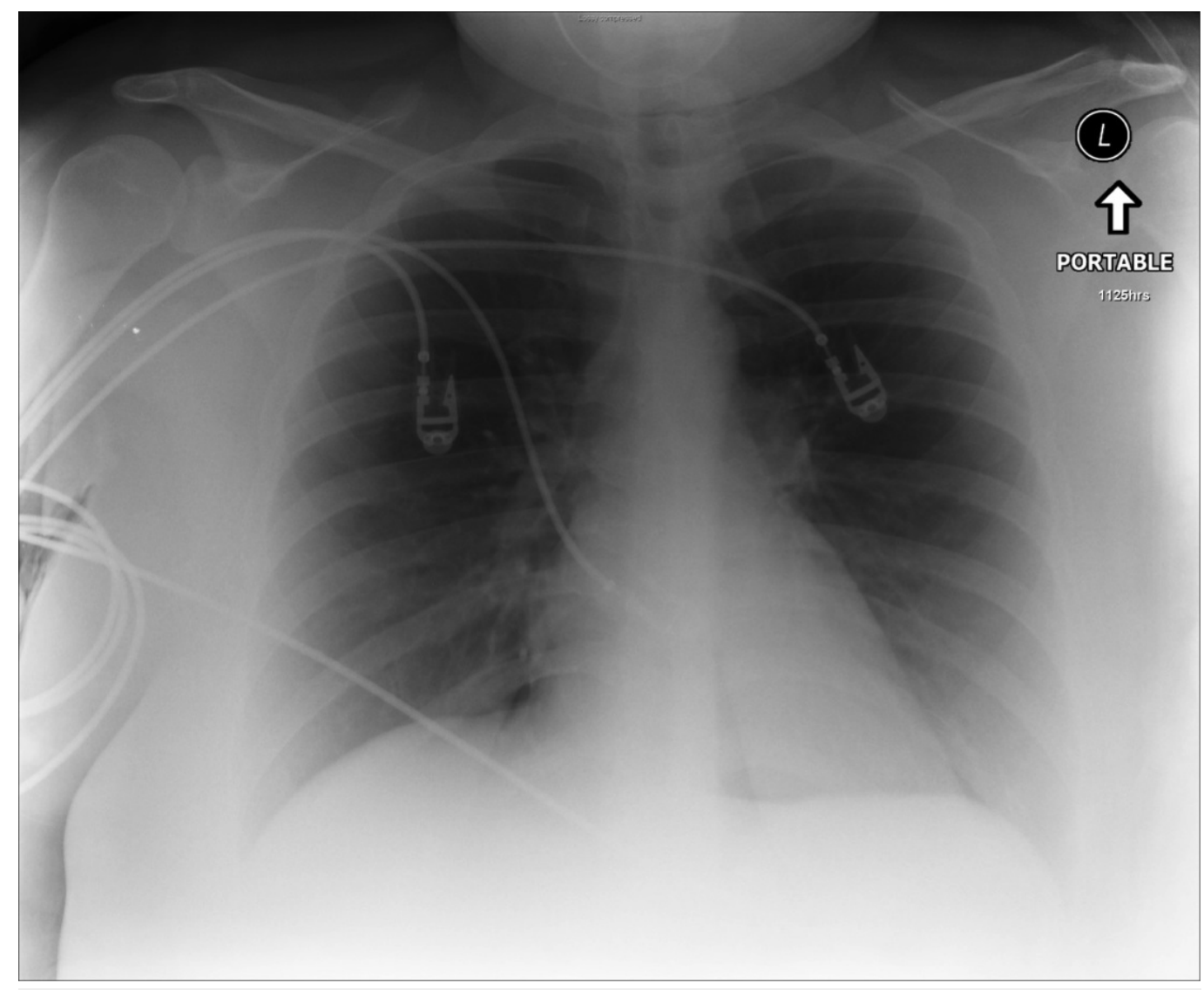

FIGURE 2: Initial CXR performed on patient presenting with acute asthma exacerbation (Source: MH Parsons) 


\section{Cureus}

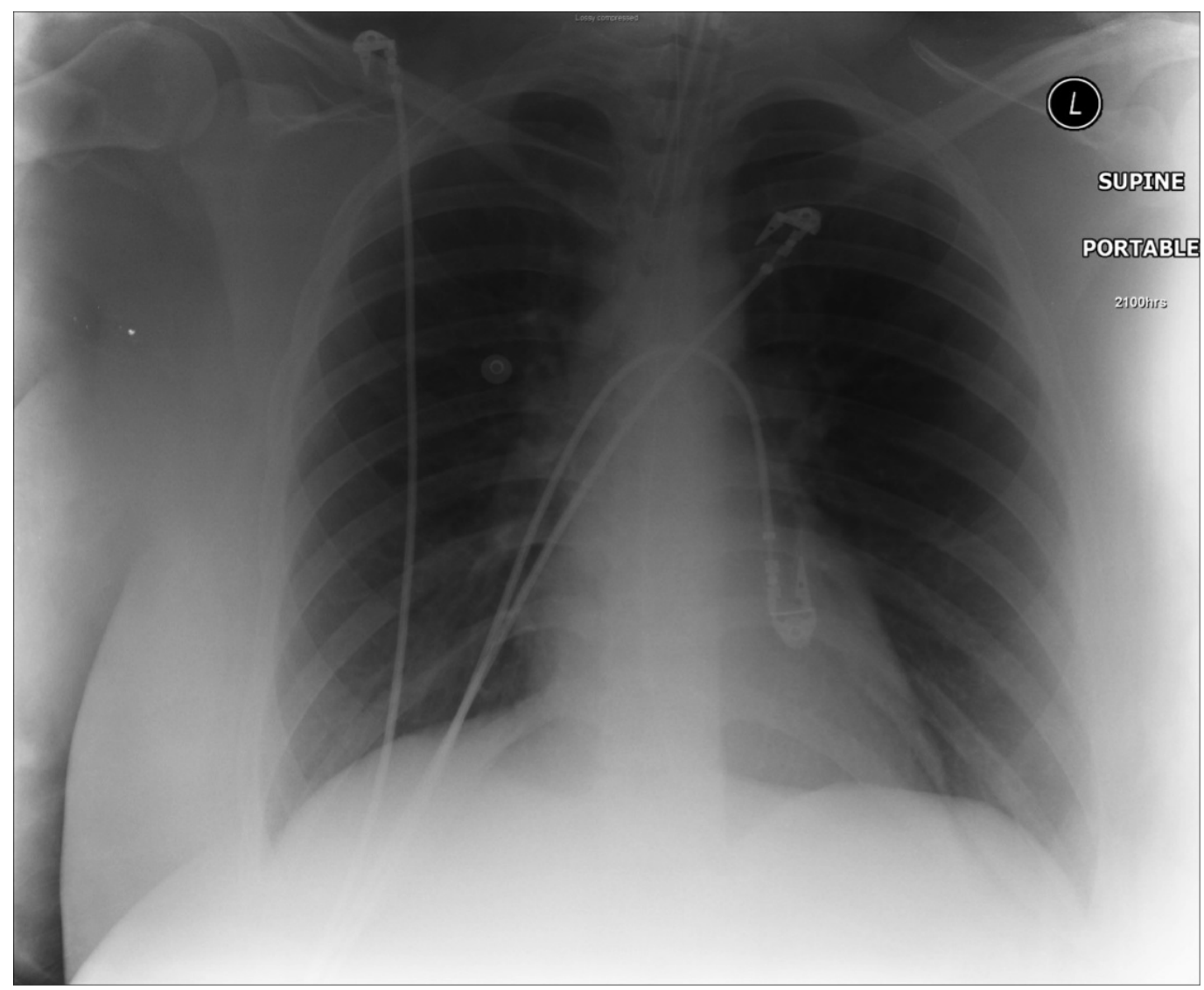

FIGURE 3: Repeat CXR post-intubation of patient presenting to the ER with acute asthma exacerbation (Source: MH Parsons)

Before conducting the session, an instructor performed a dry run of the scenario. This allowed us to identify and address technical issues that might adversely affect the learning experience. We then developed a checklist based on the scenario's learning objectives to allow us to assess the trainees during the scenario's execution. This also aided in identifying specific areas of performance to be addressed during debriefing.

During the simulation, two instructors were required. The first instructor served as the scenario director, maintaining overall control of the scenario's progression. This instructor also called for the supporting documents to be uploaded or delivered to the trainees as required by the scenario template (Table 1) (Figures 1-3). A second instructor observed trainees as they progressed through the simulation, noting the points at which critical decisions and potential errors occurred. This instructor used an assessment checklist (Table 2) in order to ensure that all errors were noted for later debriefing. Both instructors participated in the post-scenario debriefing, with the second instructor serving as lead debriefer.

Scenario Assessment Checklist

Completed

History

History of presenting illness

Allergies 


\section{Cureus}

Medications

Past Medical History

Social History

Family History

Physical

HEENT

CNS

Chest

Expected Initial Actions

Telemetry

Obtain IV access

Supplemental $\mathrm{O} 2$ given

Order EKG

Order Labs

Objective 1: Managing Acute Asthma Exacerbation

Administer nebulized salbutamol or ipratroprium/salbutamol combination

Administer MgSO4

Administer IV steroids

Consider inhaled/ IM/IV epinephrine

Consider inhaled anesthetic agent

Objective 2: Managing Respiratory Fatigue

Reexamine patient

Initiate positive-pressure ventilation

Objective 3: Managing Respiratory Failure

Reexamine patient

Initiate rapid-sequence intubation

Use appropriate agent for RSI (ketamine)

Names or performs at least 3 methods to confirm ETT placement

Objective 4: Managing Complications of RSI

Obtains repeat set of vitals post-intubation

Administers IV fluid bolus(es) for hypotension 


\section{Cureus}

Considers breath stacking as cause for hypotension

Rules out tension pneumothorax as cause of hypotension

Initiates vasopressors for refractory hypotension

Conclusion

Supportive care until ICU arrives

\section{TABLE 2: Checklist of objective criteria completed by trainee(s) to be used for formative assessment or testing purposes.}

\section{Pre-briefing}

A pre-briefing was held with the trainees before the case. Here, we addressed the fiction contract - the agreement between participants and instructors to proceed as if the simulation is real while simultaneously acknowledging it is not. We reviewed the limitations of the simulation, specifically addressing technical issues that trainees might encounter during the scenario, as well as resource availability. Finally, the trainees were advised as to whether the scenario was strictly formative or if there would be an evaluative component to the session.

\section{Case}

In this simulation case, a 23-year-old female with a history of asthma presents to the emergency department complaining of shortness of breath. She recently finished a course of prednisone. She has been admitted to the hospital/ICU on past presentations. Note that the scenario is designed such that it is easily modified to the learner's level of training. For example, it may be terminated once appropriate nebulizers and steroids are ordered (i.e., after Objective 1 in Table 1 is complete) or, if being used in a more advanced learner, the scenario may progress to include Objectives 2 and 3 with patient deterioration requiring advanced management, including airway intervention. While we have provided vital signs for each step, these are easily modified to portray a more stable patient.

The scenario is conducted in a resuscitation bay with airway equipment, resuscitation cart, and defibrillator available. Drugs available include inhalers (salbutamol, ipratropium), nebules (salbutamol, ipratropium, and budesonide), intravenous steroids, magnesium sulfate, and those required for rapid-sequence intubation and advanced cardiac life support.

Upon entering the room, the trainee is met with a patient sitting upright on a gurney, dressed in a hospital gown with no intravenous lines or cardiac monitor attached. A full set of vitals is provided via a brief triage note. The trainee is then instructed to proceed with their evaluation of the patient.

\section{Debriefing}

At the conclusion of the scenario, a formal debriefing was conducted with the trainees. To establish an environment of psychological safety conducive to learning, we revisited the confidential nature of debriefing and also reaffirmed our belief in our trainees' intelligence, commitment to doing their best, and desire to improve [5]. We subsequently conducted our debriefing using a model based on the tenets of advocacy-inquiry and frame discovery [6]. An assessment tool (Table 2) used during the scenario, was used to ensure that all the errors noted 
by the instructors were discussed during the debriefing phase.

\section{Post-scenario didactics}

After the debriefing session, we conducted a brief didactic session in which we addressed any knowledge gaps identified during the scenario and subsequent debrief. This allowed our trainees to consolidate new knowledge obtained during the simulation. We also provided our trainees with various handouts and links to websites and online articles pertinent to the recognition and management of acute asthma exacerbation [7].

\section{Discussion}

Asthma exacerbation is a problem frequently encountered in the emergency department. Although the majority of cases presenting to the emergency department do not require aggressive treatment, failure to promptly recognize and address signs of patient deterioration can lead to fatal outcomes. Thus, it is vital for trainees to develop familiarity with identifying and promptly managing those patients most at risk for deterioration.

This scenario was designed to take the learner through a series of interventions ranging from basic treatments to advanced airway management in the patient with an acute asthma exacerbation. Its learning objectives focused primarily on:

1. Managing a patient presenting with an acute asthma exacerbation.

2. Recognizing and addressing respiratory fatigue in the acute asthma patient.

3. Identifying and addressing signs of impending respiratory failure and arrest.

Given that the patient with an asthma exacerbation can present within a spectrum of severity ranging from mild shortness of breath to respiratory arrest, we intentionally designed this scenario to be easily adapted to fit the needs of a wide audience. Its stepwise algorithm can be terminated at any given point according to the learner's level of training by modifying the vital signs to reflect patient stabilization once the desired actions are undertaken by the learner.

\section{Conclusions}

The ability to identify and competently manage the patient with acute asthma exacerbation presenting to the emergency department is vital in preventing patient deterioration and possible death. Here, we describe a simulation scenario utilized to help the trainee gain familiarity with diagnosing and treating the deteriorating asthma patient, incorporating both simulation-based and didactic learning.

\section{Additional Information \\ Disclosures}

Human subjects: All authors have confirmed that this study did not involve human participants or tissue. Animal subjects: All authors have confirmed that this study did not involve animal subjects or tissue. Conflicts of interest: In compliance with the ICMJE uniform disclosure form, all authors declare the following: Payment/services info: All authors have declared that no financial support was received from any organization for the submitted work. Financial relationships: All authors have declared that they have no financial relationships at present or within the previous three years with any organizations that might have an interest in the submitted work. Other relationships: All authors have declared that there are no other 
relationships or activities that could appear to have influenced the submitted work.

\section{Acknowledgements}

This project was supported by Tuckamore Simulation Research Collaborative and Emergency Medicine Education Committee, Memorial University of Newfoundland.

\section{References}

1. Moorman J, Akinbami L, Bailey C, et al.: National Surveillance of Asthma: United States, 2001-2010. National Center for Health Statistics, Vital Health Stat. 2012, Series 3:1-67.

2. Rodrigo G, Rodrigo C, Hall J: Acute asthma in adults: A review . Chest. 2004, 125:1081-1102. 10.1378/chest.125.3.1081

3. Ilgen J, Sherbino J, Cook D: Technology-enhanced simulation in emergency medicine: a systematic review and meta-analysis. Acad Emerg Med. 2013, 20:117-27. 10.1111/acem.12076

4. Whalen D, Angus K, Dubrowski A: Propranolol Overdose: An Emergency Medicine Simulation Scenario. Cureus. 2014, 6:198. 10.7759/cureus.198

5. Rudolph J, Simon R, Raemer D, et al.: Debriefing as formative assessment: closing performance gaps in medical education. Acad Emerg Med. 2008, 15:1010-1016. 10.1111/j.1553-2712.2008.00248.x

6. Rudolph J, Simon R, Rivard P, et al: Debriefing with good judgment: Combining rigorous feedback with genuine inquiry. Anesthesiol Clin. 2007, 25:361-76.

10.1016/j.anclin.2007.03.007

7. Hodder R, Lougheed M, Rowe B, et al.: Appendix to management of acute asthma in adults in the emergency department: nonventilatory management. CMAJ. 2009, 182:55-67.

10.1503/cmaj.080072 